\title{
RIGIDITY OF COMPLETE MINIMAL HYPERSURFACES IN A HYPERBOLIC SPACE
}

\author{
Nilton M. Barroso Neto, Qiaoling Wang and Changyu Xia \\ Universidade de Brasília, Departamento de Matemática \\ 70910-900, Brasília - DF, Brazil; n.m.b.neto@mat.unb.br \\ Universidade de Brasília, Departamento de Matemática \\ 70910-900, Brasília - DF, Brazil; wang@mat.unb.br \\ Universidade de Brasília, Departamento de Matemática \\ 70910-900, Brasília - DF, Brazil; xia@mat.unb.br
}

\begin{abstract}
This paper provides a gap theorem for the first eigenvalue of the stability operator of complete immersed minimal hypersurfaces of dimension no less than five in a hyperbolic space. Namely, we show that an $n(\geq 5)$-dimensional complete immersed minimal hypersurface $M$ in a hyperbolic space is totally geodesic if the first eigenvalue of the stability operator of $M$ is bigger than some concrete constant and if the $L^{2}$ norm of the length of the second fundamental form of $M$ grows properly.
\end{abstract}

\section{Introduction}

The celebrated Bernstein theorem [2] states that the only complete minimal graphs in $\mathbf{R}^{3}$ are planes. The works of Fleming [14], De Giorgi [8], Almgren [1] and Simons [22] tell us that the Bernstein Theorem is valid for complete minimal graphs in $\mathbf{R}^{n+1}$ provided that $n \leq 7$. Counterexamples to the theorem for $n \geq 8$ were found by Bombieri-De Giorgi-Giusti [3] and later by Lawson [15]. On the other hand, it has been shown independently by do Carmo-Peng [11], Fischer ColbrieSchoen [13] that a complete stable minimal surface in $\mathbf{R}^{3}$ must be a plane. For the higher dimensional case, it is still unknown if a complete oriented stable minimal hypersurface in $\mathbf{R}^{n+1}(3 \leq n \leq 7)$ is a hyperplane. However, do Carmo and Peng have proved the following result.

Theorem A. (do Carmo and Peng [10]) Let $M^{n}$ be a complete stable minimal hypersurface in $\mathbf{R}^{n+1}$. If

$$
\lim _{R \rightarrow \infty} \frac{\int_{B_{p}(R)}|A|^{2}}{R^{2 q+2}}=0, \quad q<\sqrt{\frac{2}{n}},
$$

then $M$ is a hyperplane. Here, $B_{p}(R)$ denotes the geodesic ball of radius $R$ centered at $p \in M$ and $A$ is the second fundamental form of $M$.

Many interesting generalizations of the above do Carmo-Peng's theorem have been obtained in recent years (cf. $[9,12,18,19,20,21,23]$ etc.). In the present paper, we shall prove similar result for complete minimal hypersurfaces in a hyperbolic space.

doi:10.5186/aasfm.2015.4036

2010 Mathematics Subject Classification: Primary 53C20, 53C42. rigidity.

Key words: Minimal hypersurface, first eigenvalue, stability operator, hyperbolic space, 
By definition, the hyperbolic space $\mathbf{H}^{m}$ is a (unique) simply connected complete $m$ dimensional Riemannian manifold with a constant negative sectional curvature -1 .

Before stating our results, we recall some known facts. Let $\left(M, d s^{2}\right)$ be a complete non-compact Riemannian manifold. Let $\mu: M \rightarrow \mathbf{R}$ be a continuous function and let $\Delta$ be Laplacian operator acting on functions of $M$. We set $L_{\mu}=\Delta+\mu$ and denote by $\lambda_{1}\left(L_{\mu}, M\right)$ the first eigenvalue of $L_{\mu}$. The usual variational characterization of $\lambda_{1}\left(L_{\mu}, M\right)$ is

$$
\lambda_{1}\left(L_{\mu}, M\right)=\inf _{f \in C_{0}^{\infty}(M), f \neq 0} \frac{\int_{M}\left(|\nabla f|^{2}-\mu f^{2}\right)}{\int_{M} f^{2}},
$$

where $|\nabla f|$ denotes the magnitude of the gradient of $f$ taken with respect to $d s^{2}$. When $\mu=0$, we usually call $\lambda_{1}\left(L_{0}, M\right)$ the first eigenvalue of $M$ and denote it by $\lambda_{1}(M)$. It is well known that (cf. $[4,5,16,17]$ )

$$
\lambda_{1}\left(\mathbf{H}^{n}\right)=\frac{(n-1)^{2}}{4} .
$$

If $M$ is an $n$-dimensional complete minimal submanifold in $\mathbf{H}^{m}$, then we have (cf. [7])

$$
\lambda_{1}(M) \geq \frac{(n-1)^{2}}{4},
$$

which is equivalent to say that

$$
\int_{M}|\nabla f|^{2} \geq \frac{(n-1)^{2}}{4} \int_{M} f^{2}, \quad \forall f \in C_{0}^{\infty}(M) .
$$

If $M$ is a complete minimal hypersurface of $\mathbf{H}^{n+1}$, the stability operator of $M$ is $L_{|A|^{2}-n}$ and $M$ is said to be stable if $\lambda_{1}\left(L_{|A|^{2}-n}, M\right) \geq 0$, where $A$ is the second fundamental form of $M$ (cf. [16]). It is easy to see from (1.1) and (1.2) that the first eigenvalue of the the stability operator of a complete totally geodesic hypersurface of $\mathbf{H}^{n+1}$ is $n+\frac{(n-1)^{2}}{4}$.

In the present paper we prove a gap theorem for the first eigenvalue of the stability operator of complete minimal hypersurfaces in a hyperbolic space. Namely, we have

Theorem 1.1. Let $M$ be an $n(\geq 2)$-dimensional complete immersed minimal hypersurface in $\mathbf{H}^{n+1}$ and let $A$ be the second fundamental form of $M$. Suppose that there exists a number $q \in(0, \sqrt{2 / n})$ such that

$$
\lim _{R \rightarrow \infty} \frac{\int_{B_{p}(R)}|A|^{2}}{R^{2 q+2}}=0 .
$$

i) If $n \geq 6$ and if

$$
\lambda_{1}\left(L_{|A|^{2}-n}, M\right)>2 n-\frac{\left(2-n q^{2}\right)(n-1)^{2}}{4 n(1+q)^{2}},
$$

then $M$ is totally geodesic.

ii) If $n \leq 4$, then

$$
\lambda_{1}\left(L_{|A|^{2}-n}, M\right) \leq 2 n-\frac{\left(2-n q^{2}\right) n}{2+2 n q+n} .
$$


iii) If $n=5, q \in(0,1 / 5)$ and if

$$
\lambda_{1}\left(L_{|A|^{2}-5}, M\right)>5+\frac{25(q+1)^{2}}{10 q+7},
$$

then $M$ is totally geodesic.

iv) If $n=5$ and if $q \in[1 / 5, \sqrt{2 / 5})$, then

$$
\lambda_{1}\left(L_{|A|^{2}-5}, M\right) \leq 5+\frac{25(q+1)^{2}}{10 q+7} .
$$

In view of Theorem 1.1, it is interesting to know if a similar result for complete minimal submanifolds in a hyperbolic space holds and to study the following

Problem. What is the sharp lower bound for the first eigenvalue of the stability operator of complete minimal hypersurfaces in a hyperbolic space?

Theorem 1.1 can be generalized to complete hypersurfaces with constant mean curvature in a hyperbolic space. In order to see this, let us recall the following result.

Lemma 1.2. [19] Let $M$ be a complete non-compact immersed submanifold in a Riemannian manifold $N$. Suppose that $M$ has constant mean curvature. If there exist positive constants $\epsilon, a, b$ and $l$, such that

$$
\int_{M}|\nabla f|^{2} \geq \epsilon \int_{M} f^{2}|A|^{a}, \quad \forall f \in C_{0}^{\infty}(M),
$$

and

$$
\lim _{R \rightarrow+\infty} \frac{\int_{B_{R}(x)}|A|^{b}}{R^{l}}=0
$$

then $M^{n}$ must be minimal.

Combining Theorem 1.1 and Lemma 1.2, we immediately get

Corollary 1.3. Let $M$ be an $n(\geq 2)$-dimensional complete non-compact immersed hypersurface with constant mean curvature in $\mathbf{H}^{n+1}$ and let $A$ be the second fundamental form of $M$. Assume that there exists a number $q \in(0, \sqrt{2 / n})$ such that

$$
\lim _{R \rightarrow \infty} \frac{\int_{B_{p}(R)}|A|^{2}}{R^{2 q+2}}=0 .
$$

i) If $n \geq 6$ and if

$$
\lambda_{1}\left(L_{|A|^{2}-n}, M\right)>2 n-\frac{\left(2-n q^{2}\right)(n-1)^{2}}{4 n(1+q)^{2}},
$$

then $M^{n}$ is totally geodesic.

ii) If $n \leq 4$, then

$$
\lambda_{1}\left(L_{|A|^{2}-n}, M\right) \leq 2 n-\frac{\left(2-n q^{2}\right) n}{2+2 n q+n} .
$$

iii) If $n=5, q \in(0,1 / 5)$ and if

$$
\lambda_{1}\left(L_{|A|^{2}-5}, M\right)>5+\frac{25(q+1)^{2}}{10 q+7},
$$

then $M$ is totally geodesic. 
iv) If $n=5, q \in[1 / 5, \sqrt{2 / 5})$, then

$$
\lambda_{1}\left(L_{|A|^{2}-5}, M\right) \leq 5+\frac{25(q+1)^{2}}{10 q+7} .
$$

\section{A proof of Theorem 1.1}

In this section, we will prove the main result in this paper.

Proof of Theorem 1.1. Since $M$ is a minimal hypersurface of $\mathbf{H}^{n+1}$, we have from the Simons' formula that (cf. [6, 22])

$$
\frac{1}{2} \Delta|A|^{2}=|\nabla A|^{2}-|A|^{4}-n|A|^{2}
$$

It is well-known that (cf. [24])

$$
|\nabla A|^{2}-|\nabla| A||^{2} \geq \frac{2}{n}|\nabla| A||^{2}
$$

Recalling that $\triangle|A|^{2}=2|A| \triangle|A|+2|\nabla| A||^{2}$ and using (2.1) and (2.2) we get the following Kato-type inequality

$$
|A| \triangle|A|+|A|^{4}+n|A|^{2} \geq \frac{2}{n}|\nabla| A||^{2} .
$$

Setting

$$
\alpha=\lambda_{1}\left(L_{|A|^{2}-n}, M\right)-n,
$$

we have from the definition of $\lambda_{1}\left(L_{|A|^{2}-n}, M\right)$ that

$$
\int_{M}|\nabla f|^{2} \geq \int_{M}|A|^{2} f^{2}+\alpha \int_{M} f^{2}, \quad \forall f \in C_{0}^{\infty}(M)
$$

Setting

$$
\gamma=\frac{(n-1)^{2}}{4}
$$

we get from (1.4) that

$$
\int_{M}|\nabla f|^{2} \geq \gamma \int_{M} f^{2}, \quad \forall f \in C_{0}^{\infty}(M)
$$

Fixing an $x \in[0,1]$, we deduce from (2.5) and (2.7) that

$$
x \int_{M} f^{2}|A|^{2}+(x \alpha+(1-x) \gamma) \int_{M} f^{2} \leq \int_{M}|\nabla f|^{2}, \quad \forall f \in C_{0}^{\infty}(M) .
$$

Plugging $f|A|^{1+q}$ in (2.8) we get

$$
\begin{aligned}
& x \int_{M} f^{2}|A|^{4+2 q}+(x \alpha+(1-x) \gamma) \int_{M} f^{2}|A|^{2+2 q} \leq \int_{M}\left|\nabla\left(f|A|^{1+q}\right)\right|^{2} \\
& =(1+q)^{2} \int_{M}|A|^{2 q}|\nabla| A||^{2} f^{2}+\int_{M}|A|^{2 q+2}|\nabla f|^{2} \\
& \quad+2(1+q) \int_{M}|A|^{2 q+1} f\langle\nabla f, \nabla|A|\rangle .
\end{aligned}
$$


Multiplying (2.3) by $|A|^{2 q} f^{2}$ and integrating over $M$, we have

$$
\frac{2}{n} \int_{M}|A|^{2 q} f^{2}|\nabla| A||^{2} \leq \int_{M}|A|^{2 q+1} f^{2} \triangle|A|+\int_{M}|A|^{2 q+4} f^{2}+n \int_{M}|A|^{2 q+2} f^{2}
$$

It follows from integration by parts that

$$
\begin{aligned}
& \int_{M}|A|^{2 q+1} f^{2} \triangle|A|=-\int_{M}\left\langle\nabla\left(|A|^{2 q+1} f^{2}\right), \nabla|A|\right\rangle \\
& =-\left.(2 q+1) \int_{M}|A|^{2 q} f^{2}|\nabla| A\right|^{2}-2 \int_{M} f|A|^{2 q+1}\langle\nabla f, \nabla|A|\rangle .
\end{aligned}
$$

Multiplying (2.10) by $(1+q)$ and using (2.11), one gets

$$
\begin{aligned}
& \left.(1+q)\left(\frac{2}{n}+2 q+1\right) \int_{M}|A|^{2 q} f^{2}|\nabla| A\right|^{2} \\
& \leq(1+q) \int_{M}|A|^{2 q+4} f^{2}+(q+1) n \int_{M}|A|^{2 q+2} f^{2} \\
& \quad-2(1+q) \int_{M} f|A|^{2 q+1}\langle\nabla f, \nabla|A|\rangle .
\end{aligned}
$$

Summing up (2.9) and (2.12) we get

$$
\begin{aligned}
& x \int_{M} f^{2}|A|^{4+2 q}+(x \alpha+(1-x) \gamma) \int_{M} f^{2}|A|^{2+2 q} \\
& +\left.(1+q)\left(\frac{2}{n}+q\right) \int_{m}|A|^{2 q} f^{2}|\nabla| A\right|^{2} \\
& \leq \int_{M}|A|^{2 q+2}|\nabla f|^{2}+(1+q) \int_{M}|A|^{2 q+4} f^{2}+n(q+1) \int_{M}|A|^{2+2 q} f^{2} .
\end{aligned}
$$

For any $\epsilon>0$, we have

$$
2 \int_{M}|A|^{2 q+1} f\langle\nabla f, \nabla|A|\rangle \leq\left.\epsilon \int_{M}|A|^{2 q}|\nabla| A\right|^{2} f^{2}+\frac{1}{\epsilon} \int_{M}|A|^{2 q+2}|\nabla f|^{2} .
$$

Substituting (2.14) into (2.9), we easily obtain

$$
\begin{aligned}
& x \int_{M} f^{2}|A|^{4+2 q}+(x \alpha+(1-x) \gamma) \int_{M} f^{2}|A|^{2+2 q} \\
& \leq\left.(1+q)(1+q+\epsilon) \int_{M}|A|^{2 q}|\nabla| A\right|^{2} f^{2}+\left(1+\frac{1+q}{\epsilon}\right) \int_{M}|A|^{2 q+2}|\nabla f|^{2} .
\end{aligned}
$$

Multiplying the above inequality by $\frac{\frac{2}{n}+q}{1+q+\epsilon}$ we get

$$
\begin{aligned}
& \frac{\frac{2}{n}+q}{1+q+\epsilon}\left(x \int_{M} f^{2}|A|^{4+2 q}+(x \alpha+(1-x) \gamma) \int_{M} f^{2}|A|^{2+2 q}\right) \\
& \leq\left.(1+q)\left(\frac{2}{n}+q\right) \int_{M}|A|^{2 q}|\nabla| A\right|^{2} f^{2}+\frac{\left(\frac{2}{n}+q\right)}{\epsilon} \int_{M}|A|^{2 q+2}|\nabla f|^{2}
\end{aligned}
$$


Combining (2.13) and (2.16), we have

$$
\begin{aligned}
& \left(1+\frac{\frac{2}{n}+q}{1+q+\epsilon}\right)\left(x \int_{M} f^{2}|A|^{4+2 q}+(x \alpha+(1-x) \gamma) \int_{M} f^{2}|A|^{2+2 q}\right) \\
& \leq\left(1+\frac{\frac{2}{n}+q}{\epsilon}\right) \int_{M}|A|^{2 q+2}|\nabla f|^{2}+(1+q) \int_{M}|A|^{2 q+4} f^{2} \\
& \quad+n(q+1) \int_{M}|A|^{2+2 q} f^{2} .
\end{aligned}
$$

Now we consider different cases.

Case i): $n \geq 6$. Setting

$$
\beta=n-\frac{\left(2-n q^{2}\right)(n-1)^{2}}{4 n(1+q)^{2}},
$$

we know from (1.6) that there exists a constant $\rho>0$ such that

$$
\alpha \geq \beta+\rho \text {. }
$$

Since $q \in(0, \sqrt{2 / n})$, we can find an $\epsilon>0$ satisfying

$$
\frac{(1+q)(1+q+\epsilon)}{\frac{2}{n}+2 q+1+\epsilon}+\epsilon<1
$$

and

$$
\rho+\left(\frac{1}{\frac{(1+q)(1+q+\epsilon)}{\frac{2}{n}+2 q+1+\epsilon}+\epsilon}-1-\frac{\frac{2}{n}-q^{2}}{(1+q)^{2}}\right) \gamma>0 .
$$

Dividing (2.17) by $\left(1+\frac{\frac{2}{n}+q}{1+q+\epsilon}\right)$ and taking

$$
x=\frac{(1+q)(1+q+\epsilon)}{\frac{2}{n}+2 q+1+\epsilon}+\epsilon,
$$

one obtains that

$(2.23) \epsilon \int_{M}|A|^{2 q+4} f^{2}+(\gamma+x(\alpha-\gamma)-n x+n \epsilon) \int_{M}|A|^{2 q+2} f^{2} \leq C_{1} \int_{M}|A|^{2 q+2}|\nabla f|^{2}$,

for some positive constant $C$ depending only on $n, q, \epsilon$. It follows from (2.18), (2.19), (2.21) and (2.22) that

$$
\begin{aligned}
\gamma+x(\alpha-\gamma)-n x & =x\left(\left(\frac{1}{x}-1\right) \gamma+\alpha-n\right) \\
& \geq x\left(\left(\frac{1}{\frac{(1+q)(1+q+\epsilon)}{\frac{2}{n}+2 q+1+\epsilon}+\epsilon}-1\right) \gamma-\frac{\left(2-n q^{2}\right)(n-1)^{2}}{4 n(1+q)^{2}}+\rho\right) \\
& =x\left(\rho+\left(\frac{1}{\frac{(1+q)(1+q+\epsilon)}{\frac{2}{n}+2 q+1+\epsilon}+\epsilon}-1-\frac{\frac{2}{n}-q^{2}}{(1+q)^{2}}\right) \gamma\right)>0 .
\end{aligned}
$$


Thus, we can find an $\epsilon>0$ and a a positive constant $C_{1}$ depending only on $n, q, \epsilon, \rho$, such that

$$
\int_{M} f^{2}|A|^{2 q+4}+\int_{M} f^{2}|A|^{2 q+2} \leq C_{1} \int_{M}|A|^{2 q+2}|\nabla f|^{2}, \forall f \in C_{0}^{\infty}(M) .
$$

Recall Young's inequality

$$
a b \leq \frac{\delta^{s} a^{s}}{s}+\frac{\delta^{-t} b^{t}}{t}, \quad \frac{1}{t}+\frac{1}{s}=1,
$$

where $\delta>0$ is arbitrary and $1<t, s<+\infty$.

Setting

$$
p=\frac{2}{1+q}, \quad s=\frac{q+1}{q}, \quad t=1+q,
$$

then we have

$$
p t=2, \quad s(2 q+2-p)=4+2 q, \quad \frac{1}{t}+\frac{1}{s}=1 .
$$

It follows from Young's inequality that

$$
\begin{aligned}
|A|^{2 q+2}|\nabla f|^{2} & =f^{2}\left(|A|^{2 q+2} \frac{|\nabla f|^{2}}{f^{2}}\right) \\
& =f^{2}\left(|A|^{2 q+2-p}|A|^{p} \frac{|\nabla f|^{2}}{f^{2}}\right) \\
& \leq f^{2}\left(\frac{\delta^{s}}{s}|A|^{s(2 q+2-p)}+\frac{\delta^{t}}{t}\left(|A|^{p} \frac{|\nabla f|^{2}}{f^{2}}\right)^{t}\right) .
\end{aligned}
$$

Putting (2.25) into (2.24) we have

$$
\int_{M}|A|^{2 q+4} f^{2} \leq C_{1} \frac{q \delta^{\frac{q+1}{q}}}{q+1} \int_{M}|A|^{2 q+4} f^{2}+C_{1} \frac{\delta^{-(1+q)}}{1+q} \int_{M}|A|^{2} \frac{|\nabla f|^{2 q+2}}{f^{2 q+2}}
$$

that is,

$$
\left(1-C_{1} \frac{q \delta^{\frac{q+1}{q}}}{q+1}\right) \int_{M}|A|^{2 q+4} f^{2} \leq C_{1} \frac{\delta^{-(1+q)}}{1+q} \int_{M}|A|^{2} \frac{|\nabla f|^{2 q+2}}{f^{2 q+2}} .
$$

By choosing $\delta$ sufficiently small, we can write the above inequality as

$$
\int_{M}|A|^{2 q+4} f^{2} \leq C_{2} \int_{M}|A|^{2} \frac{|\nabla f|^{2 q+2}}{f^{2 q+2}}
$$

for a new constant $C_{2}=C_{2}(n, \epsilon, q, \rho, \delta)$.

Now, changing in (2.26) $f$ by $f^{1+q}$ we obtain

$$
\begin{aligned}
\int_{M}|A|^{2 q+4} f^{2 q+2} & \leq C_{2} \int_{M}|A|^{2} \frac{\left(\left|\nabla\left(f^{1+q}\right)\right|^{2}\right)^{1+q}}{f^{2 q(1+q)}} \\
& =C_{2}(1+q)^{2(1+q)} \int_{M}|A|^{2} \frac{f^{2 q(q+1)}|\nabla f|^{2 q+2}}{f^{2 q(q+1)}} \\
& =C_{3} \int_{M}|A|^{2}|\nabla f|^{2 q+2}
\end{aligned}
$$


Fix a $p \in M$ and choose $f$ to be a non-negative cut-off function with the properties

$$
|\nabla f| \leq \frac{1}{R}, \quad f=\left\{\begin{array}{ll}
1 & \\
0 & \text { on } M \backslash B_{p}(2 R) .
\end{array} \quad \text { on } B_{p}(R),\right.
$$

Substituting the above $f$ into (2.27) we get

$$
\int_{B_{p}(R)}|A|^{2 q+4} f^{2 q+2} \leq \int_{M}|A|^{2 q+4} f^{2 q+2} \leq C_{3} \int_{M}|A|^{2}|\nabla f|^{2 q+2} \leq C_{3} \frac{\int_{B_{p}(2 R)}|A|^{2}}{R^{2 q+2}} .
$$

Letting $R \rightarrow+\infty$ we have, by hypothesis, that the right hand side vanishes. So,

$$
\int_{M}|A|^{2 q+2}=0
$$

This implies $|A|=0$.

Case ii): $n \leq 4$. Let us assume by contradiction that

$$
\alpha>n-\frac{\left(2-n q^{2}\right) n}{2+2 n q+n} .
$$

Taking $x=1$ in (2.17), we have

$$
\begin{aligned}
& \left(1+\frac{\frac{2}{n}+q}{1+q+\epsilon}\right)\left(\int_{M} f^{2}|A|^{4+2 q}+\alpha \int_{M} f^{2}|A|^{2+2 q}\right) \\
& \leq\left(1+\frac{\frac{2}{n}+q}{\epsilon}\right) \int_{M}|A|^{2 q+2}|\nabla f|^{2}+(1+q) \int_{M}|A|^{2 q+4} f^{2} \\
& \quad+n(q+1) \int_{M}|A|^{2+2 q} f^{2} .
\end{aligned}
$$

From $q \in(0, \sqrt{2 / n})$ and $(2.29)$, we can find an $\epsilon>0$ satisfying

$$
\frac{\frac{2}{n}+q}{1+q+\epsilon}>q, \quad\left(1+\frac{\frac{2}{n}+q}{1+q+\epsilon}\right) \alpha>n(1+q) .
$$

It then follows that there exists an $\epsilon>0$ such that

$$
\int_{M} f^{2}|A|^{2 q+4}+\int_{M} f^{2}|A|^{2 q+2} \leq C_{2} \int_{M}|A|^{2 q+2}|\nabla f|^{2}, \quad \forall f \in C_{0}^{\infty}(M),
$$

for some positive constant $C_{2}$ depending only on $n, q$ and $\epsilon$. Using the same arguments as in the proof of case i) we can conclude that $M=\mathbf{H}^{n}$ and so $\alpha=\frac{(n-1)^{2}}{4}$, which contradicts to (2.29) since $n \leq 4$ and $q>0$.

Cases iii) and iv): Taking $n=5$ and $x=1$ in (2.17), we get

$$
\begin{aligned}
& \left(1+\frac{\frac{2}{5}+q}{1+q+\epsilon}\right)\left(\int_{M} f^{2}|A|^{4+2 q}+\alpha \int_{M} f^{2}|A|^{2+2 q}\right) \\
& \leq\left(1+\frac{\frac{2}{5}+q}{\epsilon}\right) \int_{M}|A|^{2 q+2}|\nabla f|^{2}+(1+q) \int_{M}|A|^{2 q+4} f^{2} \\
& \quad+5(q+1) \int_{M}|A|^{2+2 q} f^{2} .
\end{aligned}
$$


When $q \in(0, \sqrt{2 / 5})$ and

$$
\alpha>\frac{25(q+1)^{2}}{10 q+7}
$$

we can find an $\epsilon>0$ such that

$$
\frac{\frac{2}{5}+q}{1+q+\epsilon}>q, \quad\left(1+\frac{\frac{2}{5}+q}{1+q+\epsilon}\right) \alpha>5(1+q) .
$$

Thus (2.31) also holds in this case. As in the proof of case i), we know that $M$ is totally geodesic. Therefore $\alpha=4$, which, combining with (2.34), implies that $q<\frac{1}{5}$. Consequently, we know that items iii) and iv) in Theorem 1.1 hold.

\section{References}

[1] Almgren, F. J., JR.: Some interior regularity theorems for minimal surfaces and an extension of Bernstein's theorem. - Ann. of Math. (2) 84, 1966, 277-292.

[2] Bernstein, S.: Sur un théorème de géométrie et ses application aux équations aux dérivées partielles du type elliptique. - Comm. Soc. Math. Kharkov (2) 15, 1915-1917, 38-45.

[3] Bombieri, E., E. De Giorgi, and E. Giusti: Minimal cones and the Bernstein problem. Invent. Math. 7, 1969, 243-268.

[4] Chavel, I.: Riemannian geometry. A modern introduction. 2nd edition. - Cambridge Stud. Adv. Math., Cambridge Univ. Press, 2006.

[5] Cheng, S. Y.: Eigenvalue comparison theorems and its geometric applications. - Math. Z. 143, 1975, 279-297.

[6] Chern, S. S., M. P. do Carmo, and S. Kobayashi: Minimal submanifolds of a sphere with second fundamental form of constant length. - In: Functional Analysis and Related Fields, Springer, 1970, 59-75.

[7] Cheung, L. F., and P. F. Leung: Eigenvalue estimates for submanifolds with bounded mean curvature in the hyperbolic space. - Math. Z. 236, 2001, 525-530.

[8] De Giogi, E.: Una estensione del teorema di Bernstein. - Ann. Sc. Norm. Super. Pisa Cl. Sci. (3) $19,1965,79-85$.

[9] Do CARmo, M., and D. Zhou: Berstein-type theorems in hypersurfaces with constant mean curvature. - An. Acad. Brasil. Ciênc. 72, 2000, 301-310.

[10] do Carmo, M. P., and C. K. Peng: Stable complete minimal surfaces in $\mathbf{R}^{3}$ are planes. Bull. Amer. Math. Soc. (6) 1, 1979, 903-906.

[11] Do Carmo, M. P., and C. K. Peng: Stable complete minimal hypersurfaces. - Proc. Beijing Symp. Differential Equations and Differential Geometry 3, 1980, 1349-1358.

[12] Elbert, M. F., B. Nelli, and H. Rosenberg: Stable constant mean curvature hypersurfaces. - Proc. Amer. Math. Soc. 135, 2007, 3359-3366.

[13] Fisher-Colbrie, D., and R. Schoen: The structure of complete minimal surfaces in 3manifolds with non-negative scalar curvature. - Comm. Pure Appl. Math. (2) 33, 1980, 199211.

[14] Fleming, W. H.: On the oriented Plateau problem. - Rend. Circ. Math. Palermo (2) 11, 1962, 69-90.

[15] Lawson, B.: The equivariant Plateau problem and interior regularity. - Trans. Amer. Math. Soc. 173, 1972, 231-250.

[16] Li, P.: Geometric analysis. - Cambridge Stud. Adv. Math. 134, Cambridge Univ. Press, 2012. 
[17] MCKeAn, H.P.: An upper bound for the spectrum of $\Delta$ on a manifold of negative curvature. - J. Differential Geom. 6, 1970, 359-366.

[18] Nelli, B., and M. Soret: Stably embedded minimal hypersurfaces. - Math. Z. 255, 2007, $493-514$.

[19] Neto, N. M. B., and Q. Wang: Some Berstein-type rigidity theorems. - J. Math. Anal. Appl. $389,2012,694-700$.

[20] Shen, Y. B., and X. H. Zhu: On stable complete minimal hypersurfaces in $\mathbf{R}^{n+1}$. - Amer. J. Math. 120, 1998, 103-116.

[21] Shen, Y. B., and X. H. ZhU: On complete hypersurfaces with constant mean curvature and finite $L^{p}$-norm curvature in $\mathbf{R}^{n+1}$. - Acta Math. Sin. (Engl. Ser.) 21, 2005, 631-642.

[22] Simons, J.: Minimal varieties in Riemmanian manifolds. - Ann. of Math. (2) 88, 1968, 62-105.

[23] Wang, Q.: On minimal submanifolds in an Euclidean space. - Math. Nachr. 261/262, 2003, $176-180$.

[24] XIN, Y. L., and L. YANG: Curvature estimates for minimal submanifolds of higher codimension. - Chin. Ann. Math. Ser. B 30:4, 2009, 379-396.

Received 29 October $2014 \bullet$ Accepted 19 December 2014 